\title{
Molecularly Imprinted Polymers for the Detection of Food Toxins: A Minireview
}

\author{
Shivender Singh Saini, Anupreet Kaur \\ Department of Chemistry, Punjabi University, Patiala, India \\ Email:shiv_algal2007@yahoo.co.in, anu_chem06@yahoo.co.in
}

Received October 8, 2012; revised November 15, 2012; accepted December 1, 2012

\begin{abstract}
Food contamination from natural or anthropogenic sources poses severe risks to human health. It is now largely accepted that continuous exposure to low doses of food Toxins such as mycotoxins, phycotoxins can be related to several chronic diseases, including some type of cancer and serious hormonal dysfunctions. Contemporary analytical methods have the sensitivity required for contamination detection and quantification, but direct application of these methods on real samples can be rarely performed because of matrix complexity. Thus, selective analytical methods, relying on intelligent functional materials are needed. Recent years have seen the increasing use of molecular imprinted polymers in contaminant analysis in food because these materials seem to be particularly suitable for applications where analyte selectivity is essential. It offers several advantages to the agrofood industry in areas such as analysis, sensoring, extraction, or preconcentration of components. It has the potential of becoming a tool for acquiring truly simple, rapid, and robust direct measurements.
\end{abstract}

Keywords: AFB1; AFB2; AFM1; WHO; EPA; SPE; HPLC

\section{Introduction}

Food contamination due to natural toxicants can represent a significant source of food-borne illness and it poses severe risks to human health. In fact, besides the well-known food contamination due to the presence of living bacterial cells (e.g. enterotoxins from certain strains of Escherichia coli or Staphylococcus aureus), several natural contaminants represented by low mass molecules of a non-proteic nature are extremely potent acute toxins (e.g. T2 toxin) or are very strong carcinogens (e.g. aflatoxins) which are officially recognized by the World Health Organization (WHO) as biocontaminants representing a significant source of foodborne illnesses [1]. Dangerous substances in food may include natural toxicants such as mycotoxins, phycotoxins and phytotoxins [2,3], environmental contaminants such as polychlorinated dioxins [4] and polycyclic aromatic hydrocarbons [5], and chemicals such as pesticides and veterinary drugs deliberately used to increase the food supply, whose residues can be present in the processed food and potentially affect human health [6].

Contemporary analytical methods have the sensitivity required for contamination detection and quantification, but direct application of these methods on food samples can be rarely performed. Usually, contaminants are present in food at low concentration (ng/g) levels, dispersed in highly complex (thousand of different components) and morphologically structured matrices, with an elevated degree of sample-to-sample variability. Thus, such a type of matrix introduces severe disturbances, and analysis can be performed only after some clean-up and preconcentration steps [7-9].

The agricultural and food sector is in constant need of improved analytical techniques that can be used to control manufacturing processes and the safety and quality of the products $[10,11]$.

Recent years have seen a significant increase of the “molecularly imprinted solid phase extraction” (MISPE) technique in the food contaminant analysis. In fact, this technique seems to be particularly suitable for extractive applications where analyte selectivity in the presence of very complex samples represents the main problem.

MIPs have been employed in fields where a certain degree of selectivity is required such as sensors [12], chromatography [13] and catalysis [14]. However, nowadays their use in solid-phase extraction, so-called molecularly imprinted solid-phase extraction (MISPE), is by far the most advanced technical application of MIPs. Current sample pre-treatment methods, mostly based on the solid phase extraction technique, are very fast and economical. As economical, rapid and selective clean-up methods (relying on "intelligent” materials) are needed, 
solid phase extraction and clean-up methods based on molecularly imprinted polymers (molecularly imprinted solid phase extraction, MISPE) seem to represent natural candidates to circumvent the drawbacks typical of more traditional solid phase extraction techniques $[15,16]$.

\section{Molecularly Imprinted Solid-Phase Extraction}

Three different approaches to prepare MIPs have been reported: covalent, non-covalent and semi-covalent approaches. The non-covalent approach is the most widely used for the preparation of MIPs in food analysis thanks to its versatility. However, in parallel, such versatility is also the origin of some of the drawbacks attributed to MIPs. In this sense, the necessity of using a high amount of functional monomer leads to the formation of nonselective binding sites. Besides, template bleeding, overuse of certain "standard" formulations and tedious synthesis procedures are other weak points associated to this area that need to be improved. Significant attempts made during past years to improve the performance of MIPs in solid-phase extraction. There are several variables, such as kind and amount of monomer or nature of cross-linker and solvent that affects the final characteristics of the obtained materials in terms of capacity, affinity and selectivity for the target analytes.

Thus, the obtainment of the optimum MIP to be used in solid-phase extraction might take several weeks of trial-and-error experiments using different formulations. This fact has provoked an overuse of certain standard formulations (i.e. the typical 1:4:20 template:monomer: cross-linker molar ratio) [17].

\section{Applications to Food Samples}

The modern food industry is a complex and highly organized business, manufacturing a wide range of food types, including minimally processed products, modified atmosphere-packaged foods, specialist dietary formulas, and products with few or no additives. The industry is increasingly aware of the demands from the consumer for wholesome manufactured foods and, indeed, must now ensure compliance with national and international legislation on ensuring food safety and quality for the consumer. With requirements such as these, the industry is aware of the risks posed by improper control of the product quality and the resulting financial consequences. The food industry is rapidly changing its practices of end product testing (quality control) to wider quality assurance and management systems.

Analytical testing for microbiological and non-microbiological analytes must be considered in the context of a properly evaluated and implemented quality assurance system, which can be specific, rapid, and on- or nearline to cope with modern just-in-time manufacturing. From the food safety perspective, the majority of testing carried out by the agrofood industry is for microorganisms (e.g., pathogens and spoilage organisms), microbial toxins (e.g., mycotoxins and bacterial toxins), antibiotic residues, pesticides, and artificial hormones (Table 1).

As mentioned above most of the MIPs used in food analysis using SPE were prepared via noncovalent imprinting by bulk polymerization, several MISPE applications have focused on the extraction of compounds in food samples (Table 2). De Prada et al. [18] developed an on-line MISPE procedure for the selective preconcentration and voltammetric determination of sulfamethazine in milk, spiked at low concentrations.

The analytes studied included the residues of herbicides or drugs in food [19], Chapuis et al. [20] investigated the retention mechanism of analytes in MISPE by means of molecular modeling and applied MISPE to the cleanup of grape juice and soil extracts, spiked with triazines.

Maier et al. [21] studied a new analytical method for the determination of the carcinogenic mycotoxin ochratoxin in red wines, involving two-dimensional SPE cleanup on C18 silica and a target-selective MIP. Zhou et al. [22] developed an on-line MISPE-PE method for the rapid screening of OTA in wheat extracts with fluorescence detection, in which each analysis process required less than $5 \mathrm{~min}$ to complete. Moreover, LC/MS can be combined with this MISPE-PE for the rapid identification and quantification of OTA.

Blahova et al. [23] reported a MISPE method for the selective extraction of $(+)$-catechin in green tea. Under optimal conditions, a very good recovery (95\%) and selec-

Table 1. Advantages of MIPs in comparison with natural receptors.

MIPs can be prepared for practically any compound

MIPs can work in organic solvents

Polymers are compatible with microfabrication

MIPs have similar affinity as compared to natural biomoleculesbut often better specificity

MIPs are stable at low/high pHs, pressure and temperature

Polymers are inexpensive

Table 2. Food analysis.

\begin{tabular}{cc}
\hline Analyte Class & Example \\
\hline Pharmaceuticals & Antibiotics, steroids \\
Additives & Preservatives, sweeteners, colorants, flavors \\
Contaminants & Pathogenic bacteriae, microbial toxins \\
Herbicides, pesticides & Triazines \\
Minerals, trace metals & Heavy-metal ions \\
\hline
\end{tabular}


tivity were obtained by MISPE. Theodoridis and Manesiotis [24] presented the first application of caffeine MIP in SPE, allowing direct loading of aqueous samples, such as beverages and spiked human plasma.

Molinelli et al. [25] reported the first application of MISPE for complex beverage analysis, in which quercetin was selectively extracted from spiked red wine and determined by HPLC, demonstrating the potential of MISPE for rapid, selective, and cost-effective sample pretreatment. Puoci et al. [26] established a simple MISPE method for concentrating trace amounts of Sudan I from food matrices. Brambilla et al. [27] reported that the MISPE based on clenbuterol MIP was effective for the multi-residue purification of clenbuterol and aniline-like $\beta 2$-agonists, and successfully applied it to the SPE of clenbuterol from feeds, urine, and liver.

MISPE were used as for the preconcentration of additives in foods and compounds originating from foods [21-31]. This new MISPE procedure was able to remove the matrix compounds almost completely, and could be consequently used to determine triazines at concentrations below the established maximum residue limits, making the procedure suitable for monitoring these analytes in vegetable samples. MISPEs in preconcentration of analytes in various food samples (Table 3 ).

A. K. Wihlborg et al. [32] evaluate the utility of molecular imprinted polymer SPE technology for the extraction of chloramphenicol from shrimp. Because selectivity is introduced during the development of the MIP phase itself, it allows for a binding site that is sterically and chemically complementary to the target analyte.
For chloramphenicol, the SupelMIP SPE approach provided improved and significant increases in selectivity relative to a described conventional hydrophilic polymer SPE method. The SupelMIP Chloramphenicol method allowed high recoveries, above $90 \%$, low levels of interfering contaminants and very low limits of detection in the low ppt range [33].

Foodborne pathogens and microbial toxins are generally recognized to be the biggest problem in the food industry. In conjunction with other methods on the market, MIPs have the potential to become an efficient recognition element in detecting food contaminants [34-39].

In accordance to several reviews and scientific databases [40-42], in the last 10 years more than 1600 papers concerning molecular imprinting have been published worldwide. Considering the number of papers/year, molecular imprinted solid phase extraction-dedicated reviews excluded-is one of the fastest growing applications, with 223 papers published. Extraction of contaminants from food and beverage samples has been reported in 30 papers.

Here, Listeria monocytogenes and Staphylococcus aureus were employed in a surface imprinting protocol by which recognition sites were created on the surface of polyamide microcapsules for later use in binding assays. Although somewhat preliminary in nature, inasmuch as the selectivities were rather poor, the study well supports the potential of using whole cells as target species. As mentioned above, imprinting protocols using proteins as target substances are less easily developed. Nonetheless, the studies that have been published demonstrate the

Table 3. MISPEs in preconcentration of analytes in various food samples.

\begin{tabular}{|c|c|c|c|c|}
\hline Analyte & Sample & Template (T)/Monomer (M) & Detection Technique & Ref. \\
\hline Caffeine & Food sample & Caffeine & $\mathrm{LC} / \mathrm{UV}$ & [28] \\
\hline Chloramphenicol & Milk & DEAEM (M) & Square wave voltametry & [33] \\
\hline Flavonol & Olive oil & MAA( M) & Fluorescence & [34] \\
\hline Sulfamethazine & Milk & MAA(M) & Square wave voltametry & [35] \\
\hline$(+)$-Catechin & Methanolextract of green tea & $(+)$-Catechin & $\mathrm{LC} / \mathrm{UV}$ & [23] \\
\hline Ochratoxin A & Red wine & Ochratoxin A & LC/fluorescence & [21] \\
\hline Caffeine and theophylline & Green tea & Caffeine and theophylline & $\mathrm{LC} / \mathrm{UV}$ & [30] \\
\hline Sudan I & Red chili, powder spiked with Sudan I & Sudan I & $\mathrm{LC} / \mathrm{UV}$ & [26] \\
\hline Tetracycline & Fish & MAA(M) & Chemiluminescence & [36] \\
\hline Vanilin & Vanilla sugar & MAA(M) & Piezoelectric sensor & [37] \\
\hline Benzoic acid derivatives & Melissa officinalis & Sulfamethazie & LC/UV & [29] \\
\hline Atropine/scopolamine & Tablets & Atropine & LC & [38] \\
\hline Harmine, harmaline & Seeds & Harman & LC & [39] \\
\hline
\end{tabular}

DEAEM: (diethylamino)ethyl methacrylic acid, MAA: methacrylic acid. 
potential of preparing efficient MIPs toward bacterial toxins and other protein contaminants.

The most commonly tested chemical analytes include antibiotics (e.g., â-lactams and sulfonamides), mycotoxins (e.g., aflatoxins and ochratoxins), and pesticides (e.g., organophosphates and atrazine). There is also concern over the presence of degraded carrageenans (polysaccharide) in infant foods and bioavailability of vitamins. The current techniques for the analysis of polysaccharides [43], antibiotics [44], and vitamins [45] include GC, HPLC, electrophoresis, microbial assays, radioligand assays, and immunological tests. Each technique has its advantages and limitations [46]. The major limitations of the chromatographic techniques include the requirement for high technical skills, expensive equipment, and lengthy and cumbersome sample preparation, whereas microbiological assays are not always reliable, require technical expertise, and are time-consuming. ELISAs are technically simple, but the antibody reagents can show batch-to-batch variation; the assays are not wholly robust, and the commercial kits generally have limited shelf life.

A large number of studies have dealt with herbicides and pesticides, not only because these substances can be enriched in crops and cattle but also for environmental analyses. Thus, a number of studies have put forward the possibility of using the imprinted materials in, for example, sewage and wastewater analyses. In addition to basic recognition studies and imprinting protocol advancement [47-50], several applications have been developed. Thus, MIPs toward herbicides/pesticides have been used in radioligand binding assays [51-53] and in sensor devices [54].

A considerable number of studies have focused on metal ions as target species. In these cases, various metal coordination monomers have been arranged around a metal ion and subsequently been fixed during polymerization. By this method, highly selective matrices have been produced, capable of distinguishing closely related metal ions. In food analysis, the possibility of detection and quantitation of traces of contaminant heavy metals is of high value. Molecular imprinting offers a means of coupling robust and highly ion selective matrices to sensor devices. In this way, rapid analyses of trace metals may be performed.

\section{Future Prospects}

MIPs has proven to be useful as a tool in agricultural and food technology. Highly selective and robust recognition matrices produced in this way can be employed in various applications when the analysis of diverse food analytes is an issue. Given the advantages of molecularly imprinted materials such as high stability, endurance, and low cost of production, it is plausible that products based on MIPs will reach the market soon.

\section{REFERENCES}

[1] J. A. Lewis and G. R. Fenwick, "Food Contaminants: Sources and Surveillance,” In: C. S. Creaser and R. Purchase, Eds., Royal Society of Chemistry, Cambridge, 1991, pp. 1-20. doi:10.1533/9781855737846.1

[2] J. W. DeVries, M. W. Trucksess and L. S. Jackson, Eds., "Mycotoxins and Food Safety," Kluwer, New York, 2002. doi:10.1007/978-1-4615-0629-4

[3] H. P. Van Egmond, "Molecular and Life History Effects of a Natural Toxin on Herbivorus and Non-Target Soil Anthropods," Ecotoxicology, Vol. 21, No. 4, 2012, pp. 1084-1093. doi:10.1007/s10646-012-0861-z

[4] S. M. Hays and L. L. Aylward, "Dioxins Risks in Perspective: Past, Present and Future," Regulatory Toxicology and Pharmacology, Vol. 37, No. 2, 2003, pp. 202-217. doi:10.1016/S0273-2300(02)00044-2

[5] S. Moret and L. S. Conte, "Polycyclic Aromatic Hydrocarbons in Edible Fats and Oils: Occurence and Analytical Methods,” Journal of Chromatography A, Vol. 882, No. 1-2, 2000, pp. 245-253.

doi:10.1016/S0021-9673(00)00079-0

[6] J. D. G. McEvoy, “Cotamination of Animal Feeding Stuffs as a Cause of Residue in Food: A Review of Regulatory Aspects Incidence and Control," Analytica Chimica Acta, Vol. 473, No. 1-2, 2002, pp. 3-26. doi:10.1016/S0003-2670(02)00751-1

[7] M. Careri, F. Bianchi and C. Corradini, "Recent Advances in the Application of Mass Spectrometry in Food-Related Analysis,” Journal of Chromatography A, Vol. 970, No. 1-2, 2002, pp. 3-64. doi:10.1016/S0021-9673(02)00903-2

[8] P. L. Buldini, L. Ricci and J. L. Sharma, "Recent Applications of Sample Preparation Technique in Food Analysis," Journal of Chromatography A, Vol. 975, No. 1-2, 2002, pp. 47-70. doi:10.1016/S0021-9673(02)01335-3

[9] A. Juan-Garcia, G. Font and Y. Pico, "Pesticide Analysis by Capillary Electrophoresis,” Journal of Separation Science, Vol 28, No. 9-10, 2005, pp. 787-790.

[10] S. L. Wilkinson, "Eating Safely in a Dirty World,” Chemical \& Engineering News, Vol. 75, No. 5, 1997, pp. 2433. doi:10.1021/cen-v075n045.p024

[11] J. H. T. Luong, P. Bouvrette and K. B. Male, "Development and Applications of Biosensors in Food Analysis," Trends in Biotechnology, Vol. 15, No. 9, 1997, pp. 369377. doi:10.1016/S0167-7799(97)01071-8

[12] C. Malitesta, E. Mazzotta, R. A. Picca, A. Poma, I. Chianella, L. Ye and K. Haupt, "MIPs Sensors: Electrochemical Approach," Analytical and Bioanalytical Chemistry, Vol. 402, No. 5, 2004, pp. 1827-1846. doi:10.1007/s00216-011-5405-5

[13] B. Toth and G. Hovari, "Chromatograpghy, SPE and Capillary Electrophoresis, MIPs: Towards Selective Stationary Phases in Liquid Chromatography and Electrophoresis," Analytical and Bioanalytical Chemistry, Vol. 325, No. 6, 2012, pp. 267-306.

[14] C. Alexander, L. Davidson and W. Hayes, "Imprinted Polymers: Artificial Molecular Recognition Materials with Applications in Synthesis and Catalysis,” Tetrahedron, 
Vol. 59, No. 12, 2005, pp. 2025-2057. doi:10.1016/S0040-4020(03)00152-2

[15] F. Lanza and B. Sellergren, "MIPs Polymers New Tailor Made Materials for Selective Solid Phase Extraction," Advances in Chromatography, Vol. 41, No. 3, 2001, pp. 138-142.

[16] V. Pichon, F. C. Hugon, "Role of MIPs for Selective Determination of Environmental Pollutants," Analytica Chimica Acta, Vol. 622, No. 1, 2008, pp. 48-61. doi:10.1016/j.aca.2008.05.057

[17] X. Jia, H. Li, J. Luo, Q. Lu, Y. Peng, L. Shi, L. Liu, S. Du and G. Zu, "Rational Design of Core Shell MIPs Based on Computational Simulation and Doehlert Experimental Optimization: Application to the Separation of Tanshinone IIA from Salvia Miltiorrhiza Bunge,” Analytical and Bioanalytical Chemistry, Vol. 403, No. 9, 2012, pp. 26912703. doi:10.1007/s00216-012-6078-4

[18] A. G. V. De Prada, P. Martínez-Ruiz, A. J. Reviejo and J. M. Pingarrón, "Solid-Phase Molecularly Imprinted OnLine Preconcentration and Voltammetric Determination of Sulfamethazine in Milk," Analytica Chimica Acta, Vol. 539, No. 1-2, 2005, pp. 125-132. doi:10.1016/j.aca.2005.02.068

[19] C. Cacho, E. Turiel, A. Martin-Esteba, C. Perez-Conde and C. Camara, "Cleanup of Triazines in Vegetable Extracts by Molecularly-Imprinted Solid-Phase Extraction using a Propazine-Imprinted Polymer," Analytical and Bioanalytical Chemistry, Vol. 376, No. 4, 2003, pp. 491496. doi:10.1007/s00216-003-1915-0

[20] F. Chapuis, V. Pichon, F. Lanza, B. Sellergren and M. C. Hennion, "Retention Mechanism of Analytes in the SolidPhase Extraction Process Using Molecularly Imprinted Polymers-Application to the Extraction of Triazines from Complex Matrices," Journal of Chromatography B, Vol. 804, 2004, pp. 93-101. doi:10.1016/j.jchromb.2003.12.033

[21] N. M. Maier, G. Buttinger, S. Welhartizki, E. Gavioli and W. Lindner, "Molecularly Imprinted Polymer-Assisted Sample Clean-Up of Ochratoxin A from Red Wine: Merits and Limitations," Journal of Chromatography B, Vol. 804, No. 1, 2004, pp. 103-111. doi:10.1016/j.jchromb.2004.01.014

[22] C. Baggiani, L. Anfossi and C. Giovannoli, "SPE of Ochratoxin by MIPs,” Analytica Chimica Acta, Vol. 591, No. 1, 2007, pp. 29-39. doi:10.1016/j.aca.2007.01.056

[23] C. He, Y. Long, J. Pan and F. Liu, "Application of MIPs to SPE of Analytes from Real Samples,” Journal of Biochemical and Biophysical Methods, Vol. 70, No. 2, 2007, pp. 133-150. doi:10.1016/j.jbbm.2006.07.005

[24] G. Theodoridis and P. Manesiotis, "Selective Solid-Phase Extraction Sorbent for Caffeine Made by Molecular Imprinting," Journal of Chromatography A, Vol. 948, No. 1-2, 2002, pp. 163-169. doi:10.1016/S0021-9673(01)01457-1

[25] A. Molinelli, R. Weiss and B. Mizaikoff, “Advanced Solid Phase Extraction Using Molecularly Imprinted Polymers for the Determination of Quercetin in Red Wine," Journal of Agricultural and Food Chemistry, Vol. 50, No. 7, 2002, pp. 1804-1808. doi:10.1021/jf011213q
[26] H. Yan, J. Qiao, Y. Pei, T. Long, W. Ding and K. Xie, "Molecularly Imprinted Solid Phase Extraction Coupled to Liquid Chromatography for Determination of Suzan Dyes in Preserved Beancurds," Food Chemistry, Vol. 132, No. 1, 2012, pp. 649-654. doi:10.1016/j.foodchem.2011.10.105

[27] G. Brambilla, M. Fiori, B. Rizzo, V. Crescenzi and G. Masci, "Use of Molecularly Imprinted Polymers in the Solid-Phase Extraction of Clenbuterol from Animal Feeds and Biological Matrices,” Journal of Chromatography B, Vol. 759, No. 1, 2001, pp. 27-32. doi:10.1016/S0378-4347(01)00199-2

[28] K. Farrington, E. Magner and F. Regan, "Predicting the Performance of Molecularly Imprinted Polymers: Selective Extraction of Caffeine by Molecularly Imprinted Solid Phase Extraction,” Analytica Chimica Acta, Vol. 566, No. 1-2, 2006, pp. 60-68. doi:10.1016/j.aca.2006.02.057

[29] A. G. V. DePrada, A. J. Reviejo and J. M Pingarron, “A Method for the Quantification of Low Concentration Sulfamethazine Residues in Milk Based on Molecularly Imprinted Clean-Up and Surface Preconcentration at a Nafion-Modified Glassy Carbon Electrode," Journal of Pharmaceutical and Biomedical Analysis, Vol. 40, No. 2, 2006, pp. 281-286. doi:10.1016/j.jpba.2005.07.022

[30] D. Wang, S. P. Hong and K. H. Row, "Solid Extraction of Caffeine and Theophylline from Green Tea by Molecular Imprinted Polymers," Korean Journal of Chemical Engineering, Vol. 21, No. 4, 2004, pp. 853-857. doi:10.1007/BF02705530

[31] E. Martinez-Carballo, C. Gonzalez-Barreiro, S. Scharf and O. Gans, "Environmental Monitoring Study of Selected Veterinary Antibiotics in Animal Manure and Soils in Austria," Environmental Pollution, Vol. 148, No. 2, 2007, pp. 570-579. doi:10.1016/j.envpol.2006.11.035

[32] A. K. Wihlborg, B. Boyd, S. Kroonauer and C. Widstrand, MIP Technologies AB, Lund Sweden.

[33] D. Kowalski, E. Pobozy and M. Trojanowicz, "Flow Injection Preconcentration of Chlrophenicol Using MIPs for HPLC Determination in Environmental Samples," Journal of Automated Methods \& Management in Chemistry, Vol. 2011, No. 10, 2011, pp. 1-10. doi:10.1155/2011/143416

[34] J. L. Suárez-Rodriguez and M. E. Diaz-Garcia, "Flavonol Fluorcent Flow-Through Sensing Based on a Molecular Imprinted Polymers," Analytica Chimica Acta, Vol. 405, No. 1-2, 2000, pp. 67-76. doi:10.1016/S0003-2670(99)00728-X

[35] A. G. Prada, P. Martınez-Ruiz, A. J. Reviejo and J. M. Pingarron, "Solid Phase Mips On-Line Preconcentration and Volatmetric Determination of Sulfamethazine in Milk," Analytica Chimica Acta, Vol. 539, No. 1-2, 2005, pp. 125132. doi:10.1016/j.aca.2005.02.068

[36] J. Du, "Molecular Imprinted Based Chemiluminesecnce Techniques in Pharmaceutical Analysis," Current Paharmaceutical Analysis, Vol. 6, No. 1, 2010, pp. 30-38.

[37] M. Avila, M. Zougagh, A. Escarpa and A. Rios, "Supported Liquid Membrane Piezoelectric Flow Sensor with Molecularly Imprinted Polymers for the Determination of 
Vanillin in Food Samples,” Talanta, Vol. 72, No. 4, 2007, pp. 1362-1369. doi:10.1016/j.talanta.2007.01.045

[38] M. Nakamura, M. Ono, T. Nakajima, Y. Ito, T. Aketo and J. Haginaka, "Uniformly Sized Molecularly Imprinted Polymers for Atropine and Its Application to Determination of Atropine and Scopolamine in Pharmaceuticals Preparations Containing Scopolia Extract," Journal of Pharmaceutical and Biomedical Analysis, Vol. 37, No. 2, 2005, pp. 231-237. doi:10.1016/j.jpba.2004.10.017

[39] J. Xie, L. Zhu and X. Xu, “ Direct Extraction of Specific Pharmacophoric Flavonoids from Ginko Leaves,” Analytical Chemistry, Vol. 74, No. 10, 2002, pp. 2352-2360. doi:10.1021/ac015755i

[40] C. Alexander, H. S. Andersson, L. I. Andersson, R. J. Ansell, N. Kirsch, I. A. Nicholls, J. O’Mahony and M. J. Whitcombe, "Molecular Imprintring Science and Technology: A Survey of the Literature for the Years," Journal of Molecular Recognition, Vol. 19, No. 2, 2006, pp. 106-180. doi:10.1002/jmr.760

[41] Society for Molecular Imprinting Database. http://www.smi.tuberlin.de/SMlbase.htm

[42] ISIS Web of Knowledge, 2008. http://www.isinet.com

[43] Y. Yabe, T. Ninomiya, T. Tatsuno and T. Okada, "Simple Colorimetric Determination of Carrageenan in Jellies and Salad Dressings," Journal of the Association of Official Analytical Chemists, Vol. 74, No. 1, 1991, pp. 10191022.

[44] H. Oka, H. Nakazawa, K. Harada and J. D. MacNeil, "Chemical Analysis of Antibiotics Used in Agriculture," AOAC International, Arlington, 1995.

[45] G. F. M. Ball, "Water-Soluble Vitamin Assays in Human Nutrition," Chapman and Hall, London, 1994. doi:10.1007/978-1-4615-2061-0

[46] K. Oestgaard, "Enzymic Microassay for the Determination and Characterization of Alginates," Carbohydrate Polymers, Vol. 19, No. 1, 1992, pp. 51-59.
doi:10.1016/0144-8617(92)90054-T

[47] A. Aherne, C. Alexander, M. J. Payne, N. Perez and E. N. Vulfson, "Bacteria-Mediated Lithography of Polymer Surfaces," Journal of the American Chemical Society, Vol. 118, No. 36, 1996, pp. 8771-8772. doi:10.1021/ja960123c

[48] B. Sellergren, "Imprinted Dispersion Polymers: A New Class of Easily Accessible Affinity Stationary Phases," Journal of Chromatography A, Vol. 673, No. 1, 1994, pp. 133-141. doi:10.1016/0021-9673(94)87066-7

[49] J. Matsui, O. Doblhoff-Dier and T. Takeuchi, “Atrazine Selective Polymer Prepared by Molecular Imprinting Technique," Chemistry Letters, Vol. 24, No. 6, 1995, pp. 489-492. doi:10.1246/cl.1995.489

[50] J. Matsui, Y. Miyoshi and T. Takeuchi, "Fluoro Functionalized Molecularly Imprinted Polymers Selective for Herbicides," Chemistry Letters, Vol. 24, No. 11, 1995, pp. 1007-1008. doi:10.1246/cl.1995.1007

[51] M. Muldoon and L. Stanker, "Polymer Synthesis and Characterization of a Molecularly Imprinted Sorbent Assay for Atrazine,” Journal of Agricultural and Food Chemistry, Vol. 43, No. 6, 1995, pp. 1424-1427. doi:10.1021/jf00054a002

[52] M. Siemann, L. I. Andersson and K. Mosbach, "Selective Recognition of the Herbicide Atrazine by Noncovalent Molecularly Imprinted Polymers," Journal of Agricultural and Food Chemistry, Vol. 44, No. 1, 1996, pp. 141145. doi:10.1021/jf950233n

[53] J. Matsui, Y. Miyoshi, O. Doblhoff-Dier and T. Takeuchi, "A Molecularly Imprinted Synthetic Polymer Receptor Selective for Atrazine,” Analytical Chemistry, Vol. 67, No. 1, 1995, pp. 4404-4408. doi:10.1021/ac00119a032

[54] S. A. Piletsky, E. V. Piletskaya, A. V. Elgersma, A. K. Yano, I. Karube, Y. P. Parhometz and A. V. Elskaya, "Atrazine Sensing by Molecularly Imprinted Membranes," Biosensors and Bioelectronics, Vol. 10, No. 9, 1995, pp. 959-964. doi:10.1016/0956-5663(95)99233-B 